\title{
A THICK HOLLOW SPHERE COMPRESSED BY EQUAL AND OPPOSITE CONCENTRATED AXIAL LOADS: AN ASYMPTOTIC SOLUTION*
}

\author{
R. DOUGLAS GREGORY ${ }^{\dagger}$, THOMAS I. MILAC $\ddagger$, AND FREDERIC Y. M. WAN $\S$
}

\begin{abstract}
We consider an elastic hollow sphere with midsurface radius $R$ and thickness $2 h$ which is subjected to two equal and opposite concentrated loads acting at the ends of a diameter. The three-dimensional linear elasticity solution to this problem consists of (i) a narrow Saint Venant component extending a distance of order $O(h)$ from each load point, (ii) a wider "edge bending" component extending a distance of order $O(\sqrt{R h})$ from each load point, and (iii) a "membrane" component which permeates the whole sphere. Because of the stress singularities at the load points, the Saint Venant component is extremely complex and difficult to calculate. We determine the other two (outer) components of the solution without any explicit knowledge of the Saint Venant (or inner) component. This is achieved by a rigorously valid method which does not depend on any physical heuristic, such as Saint Venant's principle. Our method depends on the fact, established here for the first time, that the eigenfunctions for the spherical shell satisfy bi-orthogonality relations. We use these bi-orthogonality relations to find a two-term asymptotic approximation to the outer solution of the problem; in principle, further terms could be obtained. The leading term of this approximation corresponds to the classical thin shell solution to within $O(h / R)$. Our solution is an outer solution in the sense that it is valid outside an $O(h)$ neighborhood of the load points.

We make numerical comparisons between the predictions of our two-term refined theory, those of thin shell theory, and those of the Reissner-Wan theory (which generalizes thin shell theory by allowing for transverse shear deformation). All three solutions agree closely when $h / R \leq 1 / 20$. When $h / R=1 / 10$, however, differences become pronounced. Since our refined theory is still applicable to such moderately thick shells, the results we obtain enable us to analyze the limitations of the thin shell theory. Furthermore, we find that the predictions of the Reissner-Wan theory are considerably closer to the true values than those of classical thin shell theory.
\end{abstract}

Key words. linear elasticity, spherical shells, concentrated loads, eigenfunctions

AMS subject classification. $73 \mathrm{C} 02$

PII. S0036139997315738

1. Introduction. The axisymmetric small deformation of spherical elastic bodies subjected to concentrated loads is of theoretical importance and also of practical interest. Sternberg and Rosenthal [16] obtained the first correct three-dimensional elasticity solution of this kind by solving the problem of a solid sphere subjected to two equal and opposite concentrated loads acting at the ends of a diameter. They also showed that an earlier "solution" to this problem, due to Leutert [8], was invalid because it had singularities at the load points that were physically inadmissible.

No analogous solution exists for the problem of a hollow sphere subjected to the same loading. In principle, it would be possible to obtain this solution by beginning with the Sternberg-Rosenthal solution for the solid sphere and superposing upon it a solution for the hollow sphere corresponding to a free outer surface and an inner

* Received by the editors January 29, 1997; accepted for publication (in revised form) July 1, 1997; published electronically February 19, 1999.

http://www.siam.org/journals/siap/59-3/31573.html

$\dagger^{\dagger}$ Department of Mathematics, University of Manchester, Manchester M13 9PL, UK (doug@ ma.man.ac.uk).

${ }^{\ddagger}$ Department of Applied Mathematics, University of Washington, Seattle, WA 98195 (milac@ amath.washington.edu). The research of this author was supported by National Science Foundation Grants DMS-8904845 and DMS-9107196.

$\S$ Department of Mathematics, University of California at Irvine, Irvine, CA 92697 (wan@uci.edu). The research of this author was supported by National Science Foundation Grant DMS-8904845. 
surface subjected to a suitable distribution of surface load. The supplementary hollow sphere solution might be constructed by the method described by Lur'e [10] but would be extremely complex. This method would also give no insight into the actual structure of the whole solution. An approximate asymptotic solution having a relatively simple explicit form is therefore of considerable interest.

All existing approximate solutions are based on shell theory and are therefore restricted to the case in which the hollow sphere is thin, that is, when $h / R$ is small, where $R$ is the midsurface radius and $2 h$ is the thickness of the sphere. The simplest such solution is obtained by using the membrane theory of shells (see Flügge [2]). In addition to assuming that $h / R$ is small, this theory neglects the bending stiffness of the shell. The solution is simple but is accurate only at distances that are large compared to $\sqrt{R h}$ away from the load points. Reissner [13] applied Marguerre's shallow shell theory to a shallow spherical cap under a point load at its apex. Since bending stiffness is not assumed absent in this theory, Reissner's solution is valid in a larger region than the membrane solution, that is, at distances large compared to $h$ away from the load points. Within the context of the classical theory of thin shells, Koiter [7] was the first to give a fully correct solution ${ }^{1}$ for the complete spherical shell under equal and opposite concentrated loads at the poles. While analyzing the limitations of the existing membrane and shallow shell solutions, Koiter correctly noted that all these solutions, including his own, are at best an (outer) asymptotic solution of the exact three-dimensional elasticity problem, with an inherent error of order $O(h / R)$.

Except for this order of magnitude statement, we do not know how well the classical thin shell solution approximates the true three-dimensional elasticity solution, nor do we know the usefulness of the solution by Reissner and Wan [14], which generalizes classical thin shell theory by allowing for transverse shear deformation. The present paper offers to answer these questions by providing a relatively simple (outer) asymptotic approximation to the full three-dimensional solution, valid up to and including terms of order $O(h / R)$, with an error of order $O\left(h^{2} / R^{2}\right)$. Our solution is an outer solution in the sense that it is valid outside an $O(h)$ neighborhood of the load points.

Apart from its fundamental importance in solid mechanics, the problem of a hollow sphere subjected to concentrated loads at its poles is of considerable mathematical interest. In the neighborhood of each pole we have (i) a narrow Saint Venant component extending a distance of order $O(h)$, (ii) a wider "edge bending" component extending a distance of order $O(\sqrt{R h})$, and (iii) a "membrane" component which permeates the whole shell. Because of the stress singularities at the load points, the Saint Venant component is extremely complex and difficult to calculate. We are thus confronted with the challenging mathematical problem of determining the other two (outer) components of the solution without any explicit knowledge of the (inner) Saint Venant component. We achieve this by a rigorously valid method which does not depend on any physical heuristic, such as Saint Venant's principle.

Essentially, the outer asymptotic solution is composed of two parts, a membrane part $A_{M}\left\{\mathbf{u}^{M}, \boldsymbol{\tau}^{M}\right\}$, and a "shell" part $A_{S}\left\{\mathbf{u}^{S}, \boldsymbol{\tau}^{S}\right\}$, where the coefficients $A_{M}, A_{S}$ are to be determined. ${ }^{2}$ The coefficient $A_{M}$ is readily obtained by simple equilibrium considerations. However, the determination of $A_{S}$ is considerably more subtle. It depends on the fact, established here for the first time, that the eigenfunctions for the spherical shell satisfy certain bi-orthogonality relations; the key results are presented

\footnotetext{
${ }^{1}$ An earlier solution by Berry [1] had a multivalued displacement field.

${ }^{2}$ The full representation of the outer solution is given by equations (2.4), (2.5), which includes "shell" contributions from both poles, together with their complex conjugates.
} 
in Theorems 1 and 2. We use these bi-orthogonality relations to find an exact formula for $A_{S}$ in terms of the eigenfunction $\left\{\mathbf{u}^{S}, \boldsymbol{\tau}^{S}\right\}$, for which a two-term asymptotic approximation is already known. This yields a corresponding two-term approximation to the outer solution of our problem; in principle, further terms could be obtained. The leading term of this approximation corresponds ${ }^{3}$ to thin shell theory.

We make numerical comparisons between the predictions of our two-term refined theory, those of thin shell theory, and those of the Reissner-Wan theory for the cases in which $h / R=1 / 20$ or $1 / 10$, and $\nu=1 / 3$. When $h / R=1 / 20$, all three solutions agree closely. When $h / R=1 / 10$, however, one should not expect the thin shell solution to be accurate, but our refined theory, having an error of order $O\left(h^{2} / R^{2}\right)$ (rather than $O(h / R)$ ) should still be applicable. We find pronounced differences between the predictions of the two theories, this being attributable to the effect of the thickness. We conclude that classical thin shell theory is no longer appropriate when $h / R$ is as large as $1 / 10$. However, the Reissner-Wan theory gives values that are considerably closer to the "true" values than those of classical thin shell theory. The allowance for transverse shear deformation evidently enables the Reissner-Wan theory to be used for thicker shells than classical thin shell theory.

2. The problem and its outer solution. We consider the problem of a hollow sphere (complete spherical shell) compressed by equal and opposite concentrated loads of magnitude $F$, which are applied to the exterior surface of the sphere at opposite ends of a diameter (see Figure 1). The shell is composed of homogeneous, isotropic, elastic material with Young's modulus $E$ and Poisson's ratio $\nu$. Body forces are assumed absent, and the inner and outer spherical surfaces are assumed traction free except for the concentrated loads. We employ the small displacement, linear theory of elasticity throughout; the axial symmetry of the spherical shell, and of the loading, dictates that the shell undergoes axisymmetric deformation without torsion. Our goal is to compute two-term outer asymptotic expansions for the three-dimensional elastic fields in the spherical shell, valid away from the load points, in the limit as the dimensionless shell thickness tends to zero.

In our method of solution, we expand the required elastic field in a series of (axisymmetric) eigenfunctions first obtained by Lur'e [9]. Let the shell occupy the region $a \leq r \leq b$, where $r, \phi, \theta$ are a set of spherical polar coordinates. The concentrated loads are taken to act at the exterior "poles," $r=b, \phi=0, \pi$. The eigenfunctions are solutions of the equations of elasticity in the shell that satisfy traction free conditions on the surfaces $r=a, b$, except at points along one or both of the polar axes $\phi=0, \pi$. (We will henceforth refer to the positive (negative) polar axis as the positive (negative) $z$-axis.) We summarize relevant results concerning these eigenfunctions; further details may be found in Lur'e [9] (in Russian), Vilenskaia and Vorovich [17], Milac [11], and Gregory, Milac, and Wan [6]. Certain eigenfunctions are regular everywhere except along the negative $z$-axis. We call these cap eigenfunctions because they are sufficient to expand any axisymmetric bending state regular in the cap $a \leq r \leq b$, $0 \leq \phi \leq \alpha,(0<\alpha<\pi)$. A further set of complementary cap eigenfunctions can be obtained by reflecting the cap eigenfunctions in the plane $z=0$. The complementary cap eigenfunctions are regular everywhere except along the positive $z$-axis, and are sufficient to expand any axisymmetric bending state regular in the complementary cap, $a \leq r \leq b, \alpha \leq \phi \leq \pi$. It is evident from equilibrium considerations that the cap,

\footnotetext{
${ }^{3}$ The leading term in our asymptotic approximation is not identical to thin shell theory because thin shell theory also contains spurious "higher-order" terms that are unrelated to the true asymptotic approximation.
} 


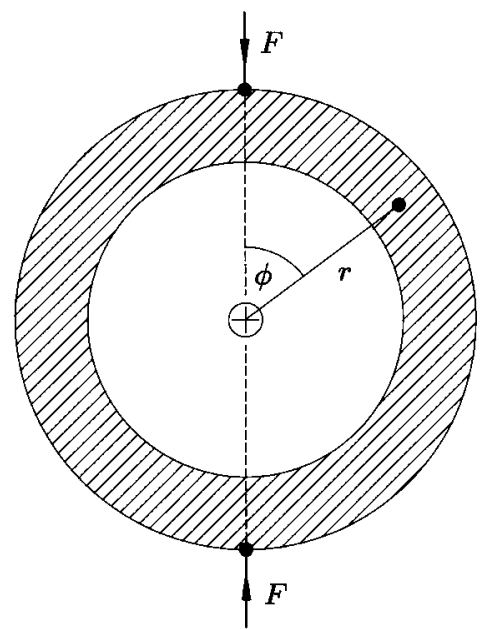

FIG. 1. The hollow sphere compressed by concentrated loads $F$ at its poles.

and complementary cap, eigenfunctions correspond to zero resultant applied load in the $z$-direction. In order to expand an elastic field that has a nonzero value of this resultant (as is clearly the case in our problem), these two sets of eigenfunctions must be supplemented by a further expansion function having a nonzero value of this resultant. The choice is not unique; we choose the state given in Appendix B because of its simple form. This additional membrane state, singular along the entire $z$-axis, completes $^{4}$ the expansion set. The only expansion function that has a simple explicit form is the membrane state. The cap and complementary cap eigenfunctions have complicated $r$-dependencies in terms of elementary functions, and $\phi$-dependencies that involve Legendre functions of complex order. The eigenvalues are the roots of the transcendental equation (A.1).

Let $h$, the half-thickness, $R$, the midsurface radius, and $\epsilon$, the dimensionless thickness of the shell, be defined by

$$
h=\frac{1}{2}(b-a), \quad R=\frac{1}{2}(b+a), \quad \epsilon=\frac{h}{R} .
$$

The cap eigenfunctions are of two types: a single shell (or S-) eigenfunction, together with its complex conjugate, and a countable infinity of Papkovich-Fadle-type (or PF) eigenfunctions. The PF-eigenfunctions are so called because, to leading order as $\epsilon \rightarrow 0$, they correspond to like-named eigenfunctions encountered in the theory of the elastic strip in plane strain. To leading order in the same limit, the S-eigenfunction corresponds to the bending solution obtained by thin shell theory. The PF-eigenfunctions are rapidly decaying in the sense that they decay exponentially away from the negative $z$-axis with decay length $O(h)$. In contrast, the S-eigenfunction has the longer decay length $O(\sqrt{R h})$ (see Appendix A). In the limit $\epsilon \rightarrow 0$, the rapid decay of the cap PF-eigenfunctions away from the negative $z$-axis implies that their contribution to the elastic fields in the complete spherical shell at some fixed latitude,

\footnotetext{
${ }^{4}$ This has not been rigorously proved, though it is widely believed to be true. As $h / R \rightarrow 0$, the leading terms of the expansion set are known to be complete for the expansion of traction or mixed data prescribed on the end of a semi-infinite strip (see Gregory [4], [5]).
} 
$\phi=\alpha(\alpha \neq 0, \pi)$, is negligible in comparison to the contribution of the cap S-eigenfunction. Similiar remarks apply to the complementary cap eigenfunctions.

We denote the cap S-eigenfunction by $\left\{\boldsymbol{\tau}^{S}, \mathbf{u}^{S}\right\}$ and the complementary cap Seigenfunction by $\left\{\boldsymbol{\tau}^{C S}, \mathbf{u}^{C S}\right\}$. Two-term asymptotic expansions for the elastic fields of $\left\{\boldsymbol{\tau}^{S}, \mathbf{u}^{S}\right\}$, valid in the limit $\epsilon \rightarrow 0$, are given in Appendix A. The corresponding expansions for the fields of $\left\{\boldsymbol{\tau}^{C S}, \mathbf{u}^{C S}\right\}$ may be found from those for $\left\{\boldsymbol{\tau}^{S}, \mathbf{u}^{S}\right\}$ by replacing $\phi$ by $\pi-\phi$ and reversing the signs of $u_{\phi}$ and $\tau_{r \phi}$; that is, by reflecting $\left\{\boldsymbol{\tau}^{S}, \mathbf{u}^{S}\right\}$ in the plane $z=0$. Two-term asymptotic expansions for the fields of the cap PF-eigenfunctions have been given by Milac [11] but are not needed here. Finally, exact expressions for the elastic fields of the membrane state, $\left\{\boldsymbol{\tau}^{M}, \mathbf{u}^{M}\right\}$, are given in Appendix B.

The exact solution to our problem, $\left\{\boldsymbol{\tau}^{E X}, \mathbf{u}^{E X}\right\}$, may thus be expanded in the form

$$
\begin{aligned}
& \boldsymbol{\tau}^{E X}=A_{M} \boldsymbol{\tau}^{M}+A_{S}\left(\boldsymbol{\tau}^{S}+\boldsymbol{\tau}^{C S}\right)+A_{S}^{*}\left(\boldsymbol{\tau}^{S}+\boldsymbol{\tau}^{C S}\right)^{*}+\boldsymbol{\tau}^{P F} \\
& \mathbf{u}^{E X}=A_{M} \mathbf{u}^{M}+A_{S}\left(\mathbf{u}^{S}+\mathbf{u}^{C S}\right)+A_{S}^{*}\left(\mathbf{u}^{S}+\mathbf{u}^{C S}\right)^{*}+\mathbf{u}^{P F}
\end{aligned}
$$

In (2.2), (2.3), $\left\{\boldsymbol{\tau}^{P F}, \mathbf{u}^{P F}\right\}$ represents an infinite sum of cap and complementary cap $\mathrm{PF}$-eigenfunctions with unknown coefficients. The fields $\left\{\boldsymbol{\tau}^{S}, \mathbf{u}^{S}\right\},\left\{\boldsymbol{\tau}^{C S}, \mathbf{u}^{C S}\right\}$ have the same coefficient $A_{S}$ because of the symmetry of $\left\{\boldsymbol{\tau}^{E X}, \mathbf{u}^{E X}\right\}$ about the plane $z=0$. Note that $A_{S},\left\{\boldsymbol{\tau}^{S}, \mathbf{u}^{S}\right\},\left\{\boldsymbol{\tau}^{C S}, \mathbf{u}^{C S}\right\}$ are complex, while $A_{M},\left\{\boldsymbol{\tau}^{M}, \mathbf{u}^{M}\right\}$ are real.

We concentrate attention on the "outer solution," $\left\{\boldsymbol{\tau}^{O}, \mathbf{u}^{O}\right\}$. This is the dominant part of the solution outside regions surrounding the load points and extending a distance $O(h)$ away from them. Because $\left\{\boldsymbol{\tau}^{P F}, \mathbf{u}^{P F}\right\}$ is composed of precisely those functions that decay away from the load points with decay length $O(h)$, the outer solution is given by

$$
\begin{aligned}
& \boldsymbol{\tau}^{O}=A_{M} \boldsymbol{\tau}^{M}+A_{S}\left(\boldsymbol{\tau}^{S}+\boldsymbol{\tau}^{C S}\right)+A_{S}^{*}\left(\boldsymbol{\tau}^{S}+\boldsymbol{\tau}^{C S}\right)^{*} \\
& \mathbf{u}^{O}=A_{M} \mathbf{u}^{M}+A_{S}\left(\mathbf{u}^{S}+\mathbf{u}^{C S}\right)+A_{S}^{*}\left(\mathbf{u}^{S}+\mathbf{u}^{C S}\right)^{*}
\end{aligned}
$$

The problem of finding the outer solution thus reduces to the determination of the two coefficients $A_{M}, A_{S}$.

3. Calculation of the coefficients $\boldsymbol{A}_{M}$ and $\boldsymbol{A}_{S}$. We determine the membrane coefficient $A_{M}$ by considering the equilibrium of a cap $a \leq r \leq b, 0 \leq \phi \leq \alpha$, which we imagine to be excised from the whole shell. Because there is no loading on the spherical surfaces of the cap other than that of the applied concentrated load, the resultant load on the cap edge at $\phi=\alpha$ must balance the load $F$ applied at the north pole; that is,

$$
2 \pi R^{2} \epsilon \int_{-1}^{1}\left[\tau_{r \phi}^{E X} \cos \alpha-\tau_{\phi \phi}^{E X} \sin \alpha\right](\sin \alpha)(1+\xi \epsilon) d \xi=F .
$$

Here, $\xi$ is a dimensionless radial coordinate defined by

$$
r=R(1+\xi \epsilon) .
$$

The S- and PF-eigenfunctions that appear in the expressions (2.2), (2.3) for $\left\{\boldsymbol{\tau}^{E X}, \mathbf{u}^{E X}\right\}$ are self-equilibrating at the cap edge and therefore make no contribution to the integral 
(3.1). Because the membrane state transverse shear stress $\tau_{r \phi}^{M} \equiv 0$, the equilibrium condition (3.1) reduces to

$$
A_{M} \sin ^{2} \alpha \int_{-1}^{1} \tau_{\phi \phi}^{M}(1+\xi \epsilon) d \xi=-\frac{F}{2 \pi R^{2}} \epsilon^{-1} .
$$

On using the expression (B.5) for $\tau_{\phi \phi}^{M}$, we find that

$$
A_{M}=-\frac{F(1+\nu)}{4 \pi E h^{2}} \epsilon,
$$

this expression being exact. Thus $A_{M}\left\{\boldsymbol{\tau}^{M}, \mathbf{u}^{M}\right\}$, the (three-dimensional) membrane contribution to the outer solution, is known exactly.

The coefficient $A_{S}$ is not so easily determined but can be found by using elastic reciprocity. For brevity, we denote the "reciprocal product" of two elastic states $\left\{\boldsymbol{\tau}^{(1)}, \mathbf{u}^{(1)}\right\},\left\{\boldsymbol{\tau}^{(2)}, \mathbf{u}^{(2)}\right\}$ over a surface $\Sigma$ by

$$
\left\langle\boldsymbol{\tau}^{(1)}, \boldsymbol{\tau}^{(2)}\right\rangle_{\Sigma}=\iint_{\Sigma}\left[\tau_{i j}^{(1)} u_{i}^{(2)}-\tau_{i j}^{(2)} u_{i}^{(1)}\right] n_{j} d S .
$$

It follows from the elastic reciprocal theorem (see Fung [3]) that if the two elastic states correspond to zero body force and are regular within a closed surface $\Sigma$, then

$$
\left\langle\boldsymbol{\tau}^{(1)}, \boldsymbol{\tau}^{(2)}\right\rangle_{\Sigma}=0
$$

In particular, it follows that

$$
\left\langle\boldsymbol{\tau}^{E X}, \boldsymbol{\tau}^{S}\right\rangle_{\Sigma}=0
$$

where $\Sigma$ is now the bounding surface of the cap $a \leq r \leq b, 0 \leq \phi \leq \alpha$ that we imagine, once again, to be excised from the spherical shell. ${ }^{5}$ Let $\mathcal{A}, \mathcal{B}$, and $\mathcal{E}$ denote the spherical inner and outer surfaces of the cap, and the cap edge at $\phi=\alpha$, respectively, so that the whole surface $\Sigma$ of the cap is

$$
\Sigma=\mathcal{A}+\mathcal{B}+\mathcal{E}
$$

Then (3.7) can we written

$$
\left\langle\boldsymbol{\tau}^{E X}, \boldsymbol{\tau}^{S}\right\rangle_{\mathcal{A}}+\left\langle\boldsymbol{\tau}^{E X}, \boldsymbol{\tau}^{S}\right\rangle_{\mathcal{B}}+\left\langle\boldsymbol{\tau}^{E X}, \boldsymbol{\tau}^{S}\right\rangle_{\mathcal{E}}=0 .
$$

Since the tractions corresponding to the elastic states $\left\{\boldsymbol{\tau}^{E X}, \mathbf{u}^{E X}\right\}$ and $\left\{\boldsymbol{\tau}^{S}, \mathbf{u}^{S}\right\}$ are zero on the inner surface of the cap, it follows that

$$
\left\langle\boldsymbol{\tau}^{E X}, \boldsymbol{\tau}^{S}\right\rangle_{\mathcal{A}}=0
$$

The reciprocal product over the cap outer surface, $\left\langle\boldsymbol{\tau}^{E X}, \boldsymbol{\tau}^{S}\right\rangle_{\mathcal{B}}$, may be written explicitly as

$$
\left\langle\boldsymbol{\tau}^{E X}, \boldsymbol{\tau}^{S}\right\rangle_{\mathcal{B}}=\iint_{\mathcal{B}}\left(\left[\tau_{r r}^{E X} u_{r}^{S}+\tau_{r \phi}^{E X} u_{\phi}^{S}\right]-\left[\tau_{r r}^{S} u_{r}^{E X}+\tau_{r \phi}^{S} u_{\phi}^{E X}\right]\right) d S .
$$

\footnotetext{
${ }^{5}$ Strictly speaking, $\boldsymbol{\tau}^{E X}$ does not satisfy the regularity condition required by (3.6) because of the singularity induced by the concentrated load $F$. We could avoid this difficulty by making a small spherical indentation in the outer surface of $\Sigma$ so as to exclude the load point. However, we take the alternative course of regarding the concentrated load as the limit of a large uniform load applied over a small patch (see (3.13)).
} 
Tractions corresponding to $\left\{\boldsymbol{\tau}^{S}, \mathbf{u}^{S}\right\}$ are zero on $\mathcal{B}$, and hence the second bracketed term in (3.11) vanishes. Because the applied load, $F$, is directed normally to the shell surface, $\tau_{r \phi}^{E X}$ also vanishes over the entire cap outer surface. The reciprocal product (3.11) therefore reduces to

$$
\left\langle\boldsymbol{\tau}^{E X}, \boldsymbol{\tau}^{S}\right\rangle_{\mathcal{B}}=\iint_{\mathcal{B}} \tau_{r r}^{E X} u_{r}^{S} d S
$$

We model the concentrated load as being applied uniformly over a small patch of the cap outer surface, the patch being centered at the pole and having angular extent $\phi_{0}$. Taking the limit $\phi_{0} \rightarrow 0$, we find that

$$
\left\langle\boldsymbol{\tau}^{E X}, \boldsymbol{\tau}^{S}\right\rangle_{\mathcal{B}}=-F u_{r}^{S}(b, 0) .
$$

Here $u_{r}^{S}(b, 0)$ means the radial displacement component of the S-eigenfunction, $\left\{\boldsymbol{\tau}^{S}, \mathbf{u}^{S}\right\}$, evaluated at the point $r=b, \phi=0$, that is, at the north pole. This simple limiting process is all that is necessary to ensure that our solution corresponds to the correct singularities at the load points.

It remains to evaluate the edge reciprocal product $\left\langle\boldsymbol{\tau}^{E X}, \boldsymbol{\tau}^{S}\right\rangle_{\mathcal{E}}$ in (3.9). Using the expansion (2.2) for $\tau^{E X}$, this product can be written

$$
\begin{gathered}
\left\langle\boldsymbol{\tau}^{E X}, \boldsymbol{\tau}^{S}\right\rangle_{\mathcal{E}}=A_{M}\left\langle\boldsymbol{\tau}^{M}, \boldsymbol{\tau}^{S}\right\rangle_{\mathcal{E}}+A_{S}\left[\left\langle\boldsymbol{\tau}^{S}, \boldsymbol{\tau}^{S}\right\rangle_{\mathcal{E}}+\left\langle\boldsymbol{\tau}^{C S}, \boldsymbol{\tau}^{S}\right\rangle_{\mathcal{E}}\right] \\
+A_{S}^{*}\left[\left\langle\left(\boldsymbol{\tau}^{S}\right)^{*}, \boldsymbol{\tau}^{S}\right\rangle_{\mathcal{E}}+\left\langle\left(\boldsymbol{\tau}^{C S}\right)^{*}, \boldsymbol{\tau}^{S}\right\rangle_{\mathcal{E}}\right]+\left\langle\boldsymbol{\tau}^{P F}, \boldsymbol{\tau}^{S}\right\rangle_{\mathcal{E}}
\end{gathered}
$$

In Theorems 1 and 2, we will show that all the reciprocal products on the right side of (3.14), except for $\left\langle\boldsymbol{\tau}^{C S}, \boldsymbol{\tau}^{S}\right\rangle_{\mathcal{E}}$, are zero. This is because the spherical shell eigenfunctions satisfy certain bi-orthogonality relations. Let $\left\{\boldsymbol{\tau}_{\lambda}, \mathbf{u}_{\lambda}\right\}$ represent any one of the cap eigenfunctions and $\left\{\boldsymbol{\tau}_{\lambda}^{C}, \mathbf{u}_{\lambda}^{C}\right\}$ the corresponding eigenfunction of the complementary cap, both corresponding to the eigenvalue $\lambda+\frac{1}{2}$. These eigenvalues satisfy (A.1) and are never real (see Appendix A and [6]). We show first the following theorem.

\section{THEOREM 1.}

$$
\left\langle\boldsymbol{\tau}^{M}, \boldsymbol{\tau}_{\lambda}\right\rangle_{\mathcal{E}}=\left\langle\boldsymbol{\tau}^{M}, \boldsymbol{\tau}_{\lambda}^{C}\right\rangle_{\mathcal{E}}=0
$$

Proof. Consider first $\left\langle\boldsymbol{\tau}^{M}, \boldsymbol{\tau}_{\lambda}\right\rangle_{\mathcal{E}}$. We observe that the value of this reciprocal product is independent of the edge $\mathcal{E}$ over which the integrals are taken, that is, independent of the angle $\alpha$. This follows immediately from the reciprocal theorem (3.6) by considering the reciprocal product $\left\langle\boldsymbol{\tau}^{M}, \boldsymbol{\tau}_{\lambda}\right\rangle$ over the surface of the spherical frustum $a \leq r \leq b, \alpha_{1} \leq \phi \leq \alpha_{2}$. Let this constant value of $\left\langle\boldsymbol{\tau}^{M}, \boldsymbol{\tau}_{\lambda}\right\rangle_{\mathcal{E}}$ be denoted by $k$. On substituting the expressions in Appendix B for $\left\{\boldsymbol{\tau}^{M}, \mathbf{u}^{M}\right\}$, we find that

$$
\begin{gathered}
k=\int_{-1}^{1}\left[E(1+\nu)^{-1}(1+\xi \epsilon)^{-1} \csc ^{2} \alpha\left(u_{\lambda}\right)_{\phi}-R\left(\cos \alpha \ln \left(\cot \frac{1}{2} \alpha\right)-1\right)\left(\tau_{\lambda}\right)_{r \phi}\right. \\
\left.+R\left(\sin \alpha \ln \left(\cot \frac{1}{2} \alpha\right)+\cot \alpha\right)\left(\tau_{\lambda}\right)_{\phi \phi}\right](\sin \alpha)(1+\xi \epsilon) d \xi .
\end{gathered}
$$

The integral (3.15) can be simplified by recalling that any cap eigenfunction, $\left\{\boldsymbol{\tau}_{\lambda}, \mathbf{u}_{\lambda}\right\}$, is self-equilibrating at the cap edge and therefore satisfies the equilibrium condition

$$
\int_{-1}^{1}\left[\left(\tau_{\lambda}\right)_{r \phi} \cos \alpha-\left(\tau_{\lambda}\right)_{\phi \phi} \sin \alpha\right](\sin \alpha)(1+\xi \epsilon) d \xi=0
$$


From (3.16) it follows that the log terms in the integrand of (3.15) cancel. With this simplification, (3.15) reduces to

$k=\int_{-1}^{1}\left[E(1+\nu)^{-1}(1+\xi \epsilon)^{-1} \csc ^{2} \alpha\left(u_{\lambda}\right)_{\phi}+R\left(\tau_{\lambda}\right)_{r \phi}+R \cot \alpha\left(\tau_{\lambda}\right)_{\phi \phi}\right](\sin \alpha)(1+\xi \epsilon) d \xi$.

Using the equilibrium condition (3.16) again, we replace the term in (3.17) containing $\left(\tau_{\lambda}\right)_{\phi \phi}$ in favor of one expressed in terms of $\left(\tau_{\lambda}\right)_{r \phi}$. On doing so, we obtain

$$
k \sin \alpha=\int_{-1}^{1}\left[E(1+\nu)^{-1}(1+\xi \epsilon)^{-1}\left(u_{\lambda}\right)_{\phi}+R\left(\tau_{\lambda}\right)_{r \phi}\right](1+\xi \epsilon) d \xi
$$

Consider now the dependence on $\alpha$ of the left and right sides of (3.18). The exact fields $\left(u_{\lambda}\right)_{\phi}$ and $\left(\tau_{\lambda}\right)_{r \phi}$ both vary with $\alpha$ as $(-\sin \alpha) P_{\lambda}^{\prime}(\cos \alpha)$, where $P_{\lambda}(\cos \phi)$ is the Legendre function of order $\lambda$ (see Appendix A). The right side of (3.18) therefore also varies with $\alpha$ as $(-\sin \alpha) P_{\lambda}^{\prime}(\cos \alpha)$. The left side of (3.18) evidently varies with $\alpha$ as $\sin \alpha, k$ being some constant independent of $\alpha$. Now $P_{\lambda}^{\prime}(\cos \alpha)$ can only be constant if $\lambda=1$ or -2 . Since $\lambda$ is known to be complex, the $\alpha$ dependencies of the left and right sides of (3.18) are incompatible unless $k=0$, which implies that $\left\langle\boldsymbol{\tau}^{M}, \boldsymbol{\tau}_{\lambda}\right\rangle_{\mathcal{E}}=0$. A similiar argument applies to $\left\langle\boldsymbol{\tau}^{M}, \boldsymbol{\tau}_{\lambda}^{C}\right\rangle_{\mathcal{E}}$, and this proves the theorem.

COROLlary 1. In particular,

$$
\left\langle\boldsymbol{\tau}^{M}, \boldsymbol{\tau}^{S}\right\rangle_{\mathcal{E}}=0
$$

Proof. This follows because $\left\{\boldsymbol{\tau}^{S}, \mathbf{u}^{S}\right\}$ is one of the cap eigenfunctions, corresponding to the particular eigenvalue $\beta$ given in (A.3).

We show next that the reciprocal product over $\mathcal{E}$ of any pair of cap or complementary eigenfunctions is zero, with the exception of the product $\left\langle\boldsymbol{\tau}_{\lambda}^{C}, \boldsymbol{\tau}_{\lambda}\right\rangle_{\mathcal{E}}$.

THEOREM 2.

(a) $\left\langle\boldsymbol{\tau}_{\lambda}, \boldsymbol{\tau}_{\mu}\right\rangle_{\mathcal{E}}=\left\langle\boldsymbol{\tau}_{\lambda}^{C}, \boldsymbol{\tau}_{\mu}^{C}\right\rangle_{\mathcal{E}}=0$ for all $\lambda, \mu$.

(b) $\left\langle\boldsymbol{\tau}_{\lambda}^{C}, \boldsymbol{\tau}_{\mu}\right\rangle_{\mathcal{E}}=0$ for $\lambda \neq \mu$.

Proof. Part (a) of the theorem is proved immediately by considering the reciprocal products $\left\langle\boldsymbol{\tau}_{\lambda}, \boldsymbol{\tau}_{\mu}\right\rangle$, or $\left\langle\boldsymbol{\tau}_{\lambda}^{C}, \boldsymbol{\tau}_{\mu}^{C}\right\rangle$, over a cap, or complementary cap, respectively. Since the contributions from the spherical surfaces $\mathcal{A}, \mathcal{B}$ are zero, it follows from the reciprocal theorem (3.6) that $\left\langle\boldsymbol{\tau}_{\lambda}, \boldsymbol{\tau}_{\mu}\right\rangle_{\mathcal{E}}=\left\langle\boldsymbol{\tau}_{\lambda}^{C}, \boldsymbol{\tau}_{\mu}^{C}\right\rangle_{\mathcal{E}}=0$.

The proof of part (b) uses a method similar to that used in the proof of Theorem 1 but is much more subtle. As in Theorem 1, we note first that the value of $\left\langle\boldsymbol{\tau}_{\lambda}^{C}, \boldsymbol{\tau}_{\mu}\right\rangle_{\mathcal{E}}$ is independent of $\alpha$. Furthermore, the $\phi$-dependencies of the eigenfunctions are expressible exactly in terms of Legendre functions. On substituting these $\phi$-dependencies into the reciprocal product and writing $x=\cos \alpha$, we find that $\left\langle\boldsymbol{\tau}_{\lambda}^{C}, \boldsymbol{\tau}_{\mu}\right\rangle_{\mathcal{E}}$ has the functional form

$$
\left\langle\boldsymbol{\tau}_{\lambda}^{C}, \boldsymbol{\tau}_{\mu}\right\rangle_{\mathcal{E}}=\left(1-x^{2}\right)\left[K_{1} P_{\lambda}(-x) P_{\mu}^{\prime}(x)+K_{2} P_{\lambda}^{\prime}(-x) P_{\mu}(x)+K_{3} x P_{\lambda}^{\prime}(-x) P_{\mu}^{\prime}(x)\right],
$$

where $K_{1}, K_{2}$, and $K_{3}$ are constants that arise when the radial portions of the eigenfunctions are integrated. Hence

$$
\left(1-x^{2}\right)\left[K_{1} P_{\lambda}(-x) P_{\mu}^{\prime}(x)+K_{2} P_{\lambda}^{\prime}(-x) P_{\mu}(x)+K_{3} x P_{\lambda}^{\prime}(-x) P_{\mu}^{\prime}(x)\right]=k,
$$

$(-1<x<1)$, where $k$ is the constant value of $\left\langle\boldsymbol{\tau}_{\lambda}^{C}, \boldsymbol{\tau}_{\mu}\right\rangle_{\mathcal{E}}$. We will show that $\left\langle\boldsymbol{\tau}_{\lambda}^{C}, \boldsymbol{\tau}_{\mu}\right\rangle_{\mathcal{E}}=0$ (when $\lambda \neq \mu$ ) by proving that (3.20) can be true only if $K_{1}=K_{2}=$ $K_{3}=0$. 
Although the equality (3.20) has been proven only for real $x \in(-1,1)$, it continues to hold for complex $z$ in any domain into which the left side may be analytically continued. Now $P_{\mu}(z)$ is analytic everywhere apart from a cut along the real interval $(-\infty,-1]$. The left side is thus evidently analytic everywhere except for cuts along the real intervals $(-\infty,-1]$ and $[1, \infty)$. However, because the left side is in fact constant, it must be continuous across these supposed cuts. We now evaluate the left side above and below the cut along the interval $[1, \infty)$ and equate the difference to zero. The result is that

$$
K_{1} P_{\lambda}(x) P_{\mu}^{\prime}(x)-K_{2} P_{\lambda}^{\prime}(x) P_{\mu}(x)+K_{3} x P_{\lambda}^{\prime}(x) P_{\mu}^{\prime}(x)=0
$$

for real $x(1<x<\infty)$. On extending (3.21) by analytic continuation, we deduce that

$$
K_{1} P_{\lambda}(z) P_{\mu}^{\prime}(z)-K_{2} P_{\lambda}^{\prime}(z) P_{\mu}(z)+K_{3} z P_{\lambda}^{\prime}(z) P_{\mu}^{\prime}(z)=0
$$

for all complex $z$ not lying on the real interval $(-\infty,-1]$.

Now in the limit as $z \rightarrow-1$,

$$
\begin{aligned}
& P_{\lambda}(z) P_{\mu}^{\prime}(z) \sim \frac{1}{\pi^{2}} \sin (\lambda \pi) \sin (\mu \pi) \frac{\ln \left(\frac{1}{2}+\frac{1}{2} z\right)}{z+1}, \\
& P_{\lambda}^{\prime}(z) P_{\mu}(z) \sim \frac{1}{\pi^{2}} \sin (\lambda \pi) \sin (\mu \pi) \frac{\ln \left(\frac{1}{2}+\frac{1}{2} z\right)}{z+1}, \\
& z P_{\lambda}^{\prime}(z) P_{\mu}^{\prime}(z) \sim-\frac{1}{\pi^{2}} \sin (\lambda \pi) \sin (\mu \pi) \frac{1}{(z+1)^{2}} .
\end{aligned}
$$

Because $z P_{\lambda}^{\prime}(z) P_{\mu}^{\prime}(z)$ grows faster than either $P_{\lambda}(z) P_{\mu}^{\prime}(z)$ or $P_{\lambda}^{\prime}(z) P_{\mu}(z)$ as $z \rightarrow-1$, it follows that (3.22) can only be satisfied if $K_{3}=0$.

With $K_{3}=0$, we now evaluate (3.22) at $z=1$ and in the limit as $|z| \rightarrow \infty$. We find that

$$
\begin{gathered}
\mu K_{1}-\lambda K_{2}=0, \\
\mu(\mu+1) K_{1}-\lambda(\lambda+1) K_{2}=0,
\end{gathered}
$$

which together imply that $K_{1}=K_{2}=0$ when $\lambda \neq \mu$. This completes the proof of part (b) of the theorem.

COROLlary 2. In particular,

(a)

$$
\left\langle\boldsymbol{\tau}^{S}, \boldsymbol{\tau}^{S}\right\rangle_{\mathcal{E}}=\left\langle\left(\boldsymbol{\tau}^{S}\right)^{*}, \boldsymbol{\tau}^{S}\right\rangle_{\mathcal{E}}=0
$$

(b)

$$
\left\langle\left(\boldsymbol{\tau}^{C S}\right)^{*}, \boldsymbol{\tau}^{S}\right\rangle_{\mathcal{E}}=0
$$

(c)

$$
\left\langle\boldsymbol{\tau}^{P F}, \boldsymbol{\tau}^{S}\right\rangle_{\mathcal{E}}=0
$$


Proof. Since $\left\{\boldsymbol{\tau}^{S}, \mathbf{u}^{S}\right\},\left\{\left(\boldsymbol{\tau}^{S}\right)^{*},\left(\mathbf{u}^{S}\right)^{*}\right\}$ are cap eigenfunctions belonging to the distinct eigenvalues $\beta, \beta^{*}$, respectively, the results (a), (b) follow from parts (a), (b) of the theorem.

Part (c) also follows from the theorem since $\left\{\boldsymbol{\tau}^{P F}, \mathbf{u}^{P F}\right\}$ is a linear combination of cap, and complementary cap, eigenfunctions corresponding to eigenvalues all different from the shell eigenvalue $\beta$.

We now return to the evaluation of the reciprocal product $\left\langle\boldsymbol{\tau}^{E X}, \boldsymbol{\tau}^{S}\right\rangle_{\Sigma}$ in (3.7). It follows from (3.10), (3.13), and (3.14), and Theorems 1 and 2, that

$$
0=\left\langle\boldsymbol{\tau}^{E X}, \boldsymbol{\tau}^{S}\right\rangle_{\Sigma}=-F u_{r}^{S}(b, 0)+A_{S}\left\langle\boldsymbol{\tau}^{C S}, \boldsymbol{\tau}^{S}\right\rangle_{\mathcal{E}}
$$

The first term on the right of (3.28) is the contribution to $\left\langle\boldsymbol{\tau}^{E X}, \boldsymbol{\tau}^{S}\right\rangle_{\Sigma}$ from the outer surface $\mathcal{B}$, the contribution from the inner surface $\mathcal{A}$ being zero. The second term is the only surviving contribution from the edge $\mathcal{E}$ (see (3.14)). Solving for $A_{S}$, we find that

$$
A_{S}=\frac{F u_{r}^{S}(b, 0)}{\left\langle\boldsymbol{\tau}^{C S}, \boldsymbol{\tau}^{S}\right\rangle_{\mathcal{E}}}
$$

Since this formula is exact, the accuracy to which the coefficient $A_{S}$ can be found depends only upon the accuracy to which the S-eigenfunction $\left\{\boldsymbol{\tau}^{S}, \mathbf{u}^{S}\right\}$ has been determined. In our refined theory we use the two-term asymptotic approximation to $\left\{\boldsymbol{\tau}^{S}, \mathbf{u}^{S}\right\}$ given in Appendix A, from which we obtain

$$
\begin{gathered}
u_{r}^{S}(b, 0)=R\left[2-2 \nu\left(1+\frac{i \omega^{2}}{1-\nu}\right) \epsilon+O\left(\epsilon^{2}\right)\right], \\
\left\langle\boldsymbol{\tau}^{C S}, \boldsymbol{\tau}^{S}\right\rangle_{\mathcal{E}}=-\frac{64}{3} E R^{3} \cos (\pi \beta) \epsilon^{2}\left[\frac{2 i \omega^{2}}{1-\nu^{2}}+\frac{7+3 \nu}{5(1-\nu)} \epsilon+O\left(\epsilon^{2}\right)\right]
\end{gathered}
$$

as $\epsilon \rightarrow 0$. In principle, further terms in these expansions could be obtained.

On combining these results in (3.29), we find that

$$
A_{S}=\frac{F}{16 E h^{2} \cos (\pi \beta)}\left[i \omega^{2}+\frac{1}{20}\left(9 \nu^{2}-5 \nu-14-20 i \nu \omega^{2}\right) \epsilon+O\left(\epsilon^{2}\right)\right] .
$$

Equations (3.4) and (3.32) determine the coefficients $A_{M}, A_{S}$ that are required to generate the outer solution $\left\{\boldsymbol{\tau}^{O}, \mathbf{u}^{O}\right\}$ correct to two terms.

4. Results. Using the coefficients $A_{M}$ and $A_{S}$ computed in the last section, and the elastic field expansions given in Appendices A and B, we can find an asymptotic approximation to the outer solution $\left\{\boldsymbol{\tau}^{O}, \mathbf{u}^{O}\right\}$, valid to two terms in the limit as $\epsilon \rightarrow 0$.

An interesting feature of the solution is that the membrane contribution $A_{M}\left\{\boldsymbol{\tau}^{M}, \mathbf{u}^{M}\right\}$ is known exactly. This is the dominant part of the solution at distances from the load points that are large compared to $\sqrt{R h}$. For instance, the important circumferential stress $A_{M} \tau_{\theta \theta}^{M}$ is given by

$$
A_{M} \tau_{\theta \theta}^{M}(\xi, \phi)=\frac{F \csc ^{2} \phi}{4 \pi R h(1+\xi \epsilon)}
$$

where $\xi$ is the dimensionless radial coordinate defined by $r=R(1+\xi \epsilon)$. The corresponding "thin shell" value is obtained by setting $\epsilon=0$. The stress (4.1) is largest 


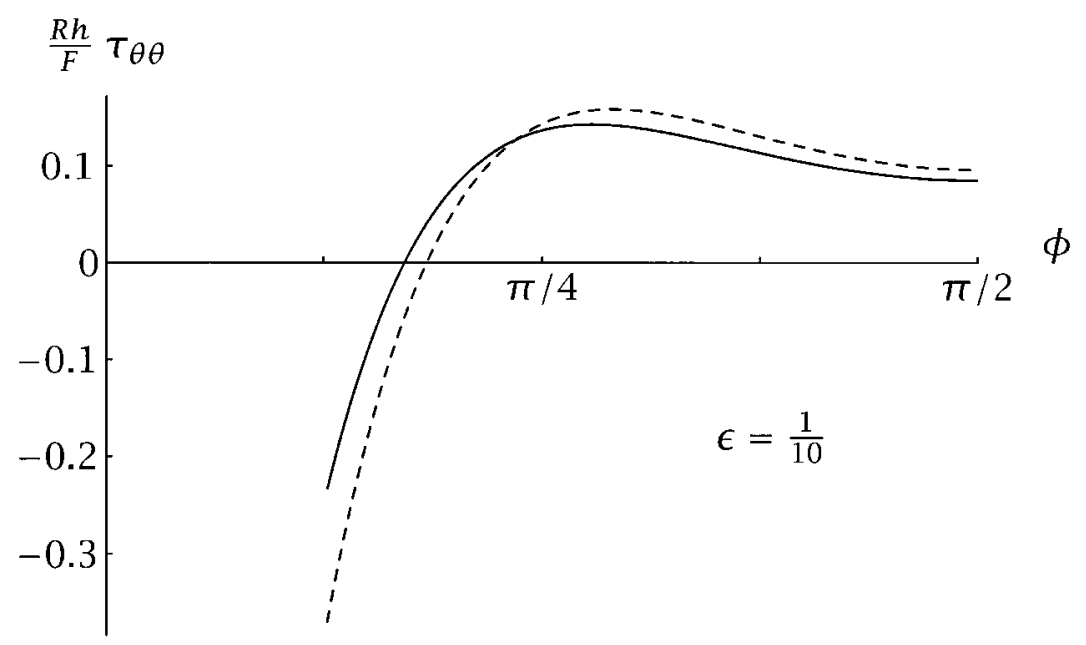

FIG. 2. The circumferential stress $\tau_{\theta \theta}$ at the outer surface of the sphere, plotted against $\phi$, for the case $\epsilon=1 / 10$ and $\nu=1 / 3$. The solid line refers to the refined theory and the dashed line to thin shell theory.

when $\xi=-1$, that is, on the inner surface of the shell, where it exceeds the thin shell value by the factor $(1-\epsilon)^{-1}$. When $\epsilon=1 / 10$, this is about $11 \%$ higher than the thin shell value.

At distances from the load points that are large compared to $h$, but not large compared to $\sqrt{R h}$, the contribution of the S-eigenfunction is significant and must be included. The solution then involves the fields in Appendix A. As an illustration, we show, in Figure 2, the variation of the circumferential stress $\tau_{\theta \theta}$ on the outer surface of the sphere, plotted against the angle $\phi$, together with the corresponding thin shell result, for the case in which $\epsilon=1 / 10$ and $\nu=1 / 3$. The two theories give significantly different results. We regard the results from our refined theory to be the more accurate since one should not expect the thin shell theory to be appropriate for a sphere of this thickness. (We do not continue our graphs up to $\phi=0$. This is because shell theories (of any order) neglect the contribution of the PF-eigenfunctions and so are only accurate at distances from the load points that are large compared to $h$. Thus $\phi$ is restricted to be large compared to $\epsilon$.)

Since the three-dimensional stress fields are rather complex, we also present formulae for the stress resultants and couples, defined by

$$
\begin{gathered}
Q_{\phi}=R \epsilon \int_{-1}^{1} \tau_{r \phi}(\xi, \phi)(1+\xi \epsilon) d \xi, \\
N_{\phi \phi}=R \epsilon \int_{-1}^{1} \tau_{\phi \phi}(\xi, \phi)(1+\xi \epsilon) d \xi, \\
M_{\phi \phi}=R^{2} \epsilon^{2} \int_{-1}^{1} \tau_{\phi \phi}(\xi, \phi) \xi(1+\xi \epsilon) d \xi,
\end{gathered}
$$

together with similar expressions for $N_{\theta \theta}, M_{\theta \theta}$. These are as follows. 


\section{Stress resultants.}

$$
\begin{aligned}
& R F^{-1} Q_{\phi}=- \frac{1}{4} \Re\left(\Lambda_{2}\right) \epsilon^{-1 / 2} \\
&+ \frac{1}{40}\left[10 \nu \Re\left(\Lambda_{2}\right)+\omega^{-2}(3+2 \nu)(2-3 \nu) \Im\left(\Lambda_{2}\right)\right] \epsilon^{1 / 2}+O\left(\epsilon^{3 / 2}\right) . \\
& R F^{-1} N_{\phi \phi}=-\frac{1}{2 \pi} \csc ^{2} \phi-\frac{1}{4} \cot \phi \Re\left(\Lambda_{2}\right) \epsilon^{-1 / 2} \\
&+ \frac{\cot \phi}{40}\left[10 \nu \Re\left(\Lambda_{2}\right)+\omega^{-2}(3+2 \nu)(2-3 \nu) \Im\left(\Lambda_{2}\right)\right] \epsilon^{1 / 2}+O(\epsilon) . \\
& R F^{-1} N_{\theta \theta}=\frac{1}{2 \pi} \csc ^{2} \phi-\frac{1}{2} \omega^{2} \Im\left(\Lambda_{1}\right) \epsilon^{-1}+\frac{1}{4} \cot \phi \Re\left(\Lambda_{2}\right) \epsilon^{-1 / 2} \\
&+\frac{1}{20}\left[\left(12 \nu^{2}+5 \nu-7\right) \Re\left(\Lambda_{1}\right)+10 \nu \omega^{2} \Im\left(\Lambda_{1}\right)\right] \\
&-\frac{\cot \phi}{40}\left[10 \nu \Re\left(\Lambda_{2}\right)+\omega^{-2}(3+2 \nu)(2-3 \nu) \Im\left(\Lambda_{2}\right)\right] \epsilon^{1 / 2}+O(\epsilon) .
\end{aligned}
$$

\section{Stress couples.}

$$
\begin{gathered}
F^{-1} M_{\phi \phi}=-\frac{1}{4} \Re\left(\Lambda_{1}\right)-\frac{1}{6} \omega^{2}(1+\nu)^{-1} \cot \phi \Im\left(\Lambda_{2}\right) \epsilon^{1 / 2} \\
+\frac{1}{40}\left[10 \nu \Re\left(\Lambda_{1}\right)+\omega^{-2}\left(1-6 \nu^{2}\right) \Im\left(\Lambda_{1}\right)\right] \epsilon+\frac{\nu \cot \phi}{40}\left[4 \Re\left(\Lambda_{2}\right)+5 \omega^{-2}(1-\nu) \Im\left(\Lambda_{2}\right)\right] \epsilon^{3 / 2}+O\left(\epsilon^{2}\right) . \\
F^{-1} M_{\theta \theta}=-\frac{1}{4} \nu \Re\left(\Lambda_{1}\right)+\frac{1}{6} \omega^{2}(1+\nu)^{-1} \cot \phi \Im\left(\Lambda_{2}\right) \epsilon^{1 / 2} \\
+\frac{\nu}{40}\left[10 \nu \Re\left(\Lambda_{1}\right)+\omega^{-2}\left(7-12 \nu^{2}\right) \Im\left(\Lambda_{1}\right)\right] \epsilon-\frac{\nu \cot \phi}{40}\left[4 \Re\left(\Lambda_{2}\right)+5 \omega^{-2}(1-\nu) \Im\left(\Lambda_{2}\right)\right] \epsilon^{3 / 2}+O\left(\epsilon^{2}\right) .
\end{gathered}
$$

In the above formulae,

$$
\begin{aligned}
& \Lambda_{1}(\phi, \beta)=\left[P_{\beta-\frac{1}{2}}(\cos \phi)+P_{\beta-\frac{1}{2}}(-\cos \phi)\right] \sec \pi \beta, \\
& \Lambda_{2}(\phi, \beta)=\epsilon^{1 / 2} \frac{\partial}{\partial \phi} \Lambda_{1}(\phi, \beta),
\end{aligned}
$$

where $\beta(\epsilon, \nu)$ is the shell eigenvalue given by (A.3). In accordance with the reflective symmetry of the elastic field about the equatorial plane $\phi=\pi / 2, \Lambda_{1}$ is an even function of $\phi$ about $\phi=\pi / 2$, while $\Lambda_{2}$ is odd. Without losing generality, we may therefore restrict $\phi$ to lie in the interval $0<\phi \leq \pi / 2$. 


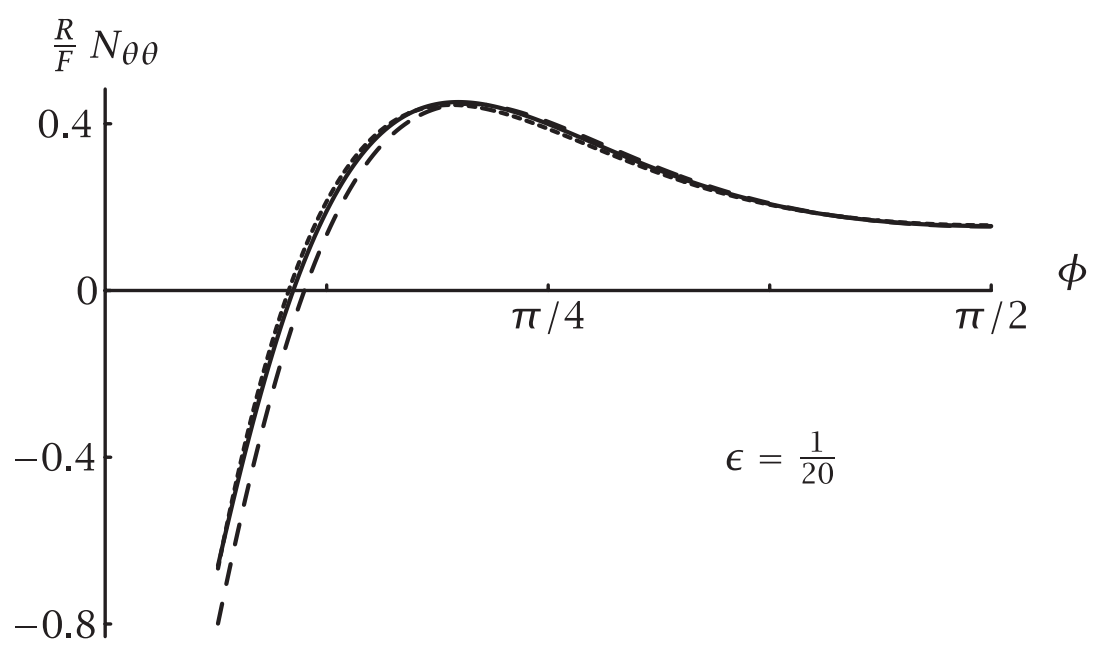

FIG. 3. The stress resultant $N_{\theta \theta}$, plotted against $\phi$, when $\epsilon=1 / 20$ and $\nu=1 / 3$. The solid line refers to the refined theory, the long-dashed line to thin shell theory, and the short-dashed line to the Reissner-Wan thin shell theory.

It should be noted that, if $\phi$ is allowed to approach the load points as $\epsilon \rightarrow$ 0 , it is not permissible to use the "nonuniform" asymptotic expansion (A.26) for the Legendre function $P_{\beta-\frac{1}{2}}(\cos \phi)$. It is permissible, however, to use the uniform asymptotic expansions given, for instance, by Olver [12, pp. 463-473]. It follows from these expansions that $\Lambda_{1}, \Lambda_{2}$ are not exponentially small as $\epsilon \rightarrow 0$ (as they would be if $\phi$ were bounded away from $0, \pi$ ), but in fact have the asymptotic form

$$
\begin{gathered}
\Lambda_{1} \sim i\left(\frac{\phi}{\sin \phi}\right)^{\frac{1}{2}} H_{0}(\beta \phi), \\
\Lambda_{2} \sim-i \epsilon^{1 / 2} \beta\left(\frac{\phi}{\sin \phi}\right)^{\frac{1}{2}} H_{1}(\beta \phi)
\end{gathered}
$$

as $\epsilon \rightarrow 0$, where $H_{n}$ is the Hankel function of the first kind (that is, $H_{n}=J_{n}+i Y_{n}$ ). In particular, it follows that, if $\phi$ is restricted so that $\epsilon^{-1 / 2} \phi$ is bounded away from zero, then

$$
\begin{aligned}
& \Lambda_{1}=O(1), \\
& \Lambda_{2}=O(1)
\end{aligned}
$$

as $\epsilon \rightarrow 0$ uniformly in $\phi$. Consequently, the error terms in the formulae (4.5)-(4.9) are also uniformly valid in $\phi$ (as $\epsilon \rightarrow 0$ ) provided that $\epsilon^{-1 / 2} \phi$ is bounded away from zero. (This is equivalent to the requirement that the distance of the field point from the load point should not be small compared to $\sqrt{R h}$.)

These results are illustrated in Figures 3-6, where we show the variation of the stress resultant $N_{\theta \theta}$ and stress couple $M_{\theta \theta}$, against $\phi$, for the cases in which $\epsilon=$ $1 / 20$ or $1 / 10$ and $\nu=1 / 3$. In each case, the predictions of our two-term refined theory are compared with those of classical thin shell theory and also those of the ReissnerWan thin shell theory, which allows for the effect of transverse shear deformation. The 


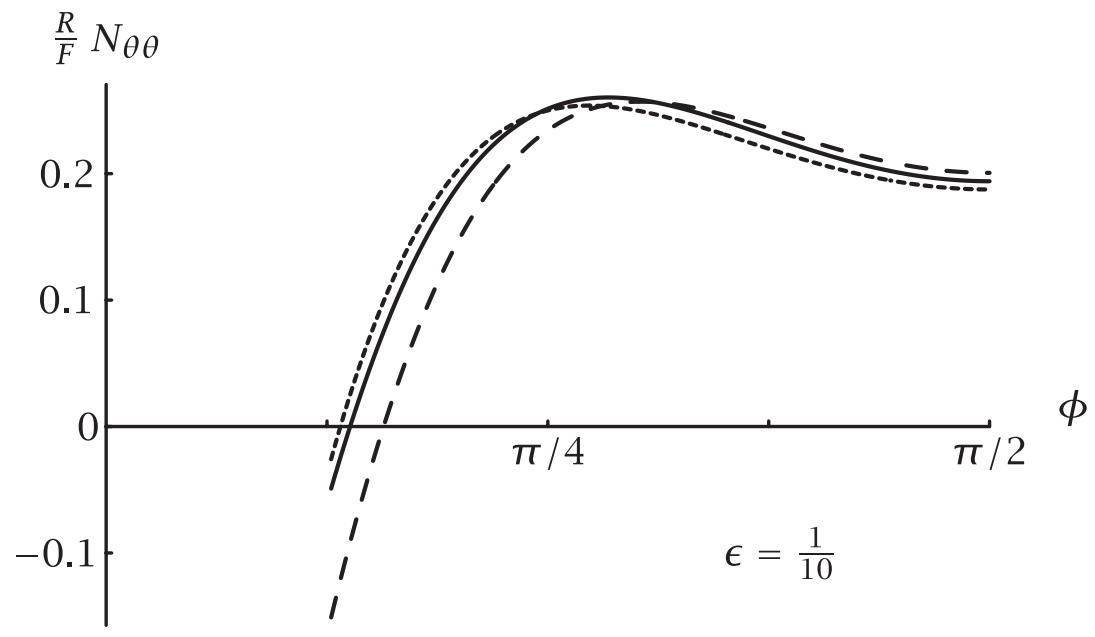

FIG. 4. The stress resultant $N_{\theta \theta}$, plotted against $\phi$ when $\epsilon=1 / 10$ and $\nu=1 / 3$. The solid line refers to the refined theory, the long-dashed line to thin shell theory, and the short-dashed line to the Reissner-Wan thin shell theory.

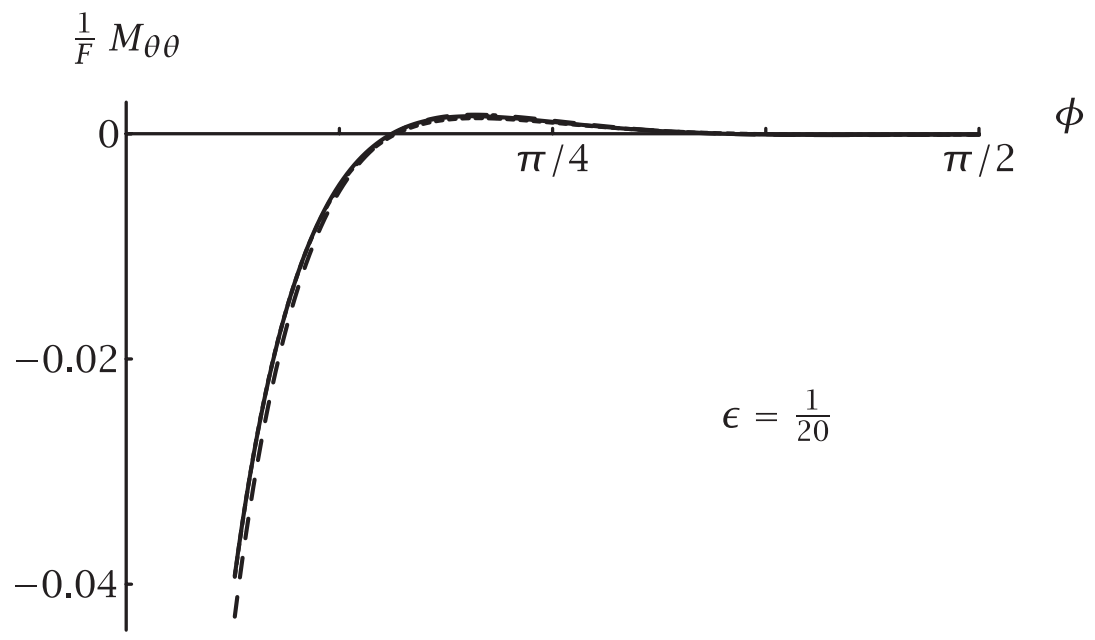

FIG. 5. The stress couple $M_{\theta \theta}$, plotted against $\phi$, when $\epsilon=1 / 20$ and $\nu=1 / 3$. The solid line refers to the refined theory, the long-dashed line to thin shell theory, and the short-dashed line to the Reissner-Wan thin shell theory. (The refined and Reissner-Wan lines are virtually coincident.)

Reissner-Wan theory (see [14]) contains ${ }^{6}$ an additional elastic constant $A_{Q}$ which, for the isotropic case considered here, takes the value $A_{Q}=6(1+\nu) / 5 E h$. The ReissnerWan graphs in Figures 3-6 were generated by substituting this value for $A_{Q}$ into the solution derived in [14]. The thin shell graphs were obtained by simply setting $A_{Q}=0$ in the same solution. For both $N_{\theta \theta}$ and $M_{\theta \theta}$ there is close agreement between all three solutions when $\epsilon=1 / 20$. When $\epsilon=1 / 10$, however, the differences between the refined and thin shell solutions are pronounced. Since the refined theory values have a relative error of order $O\left(\epsilon^{2}\right)$, one would expect this error to be negligible when $\epsilon=1 / 10$. Hence

\footnotetext{
${ }^{6}$ Actually, the Reissner-Wan theory contains a further elastic constant $D_{P}$, which is associated with couple stresses. This we set equal to zero.
} 


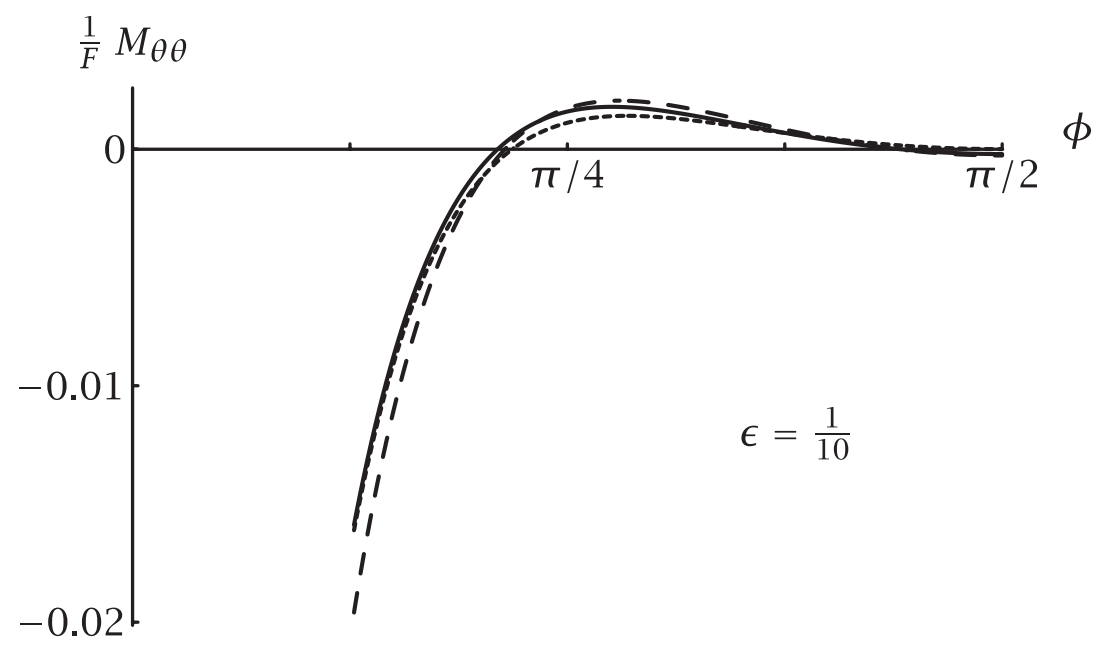

FIG. 6. The stress couple $M_{\theta \theta}$, plotted against $\phi$, when $\epsilon=1 / 10$ and $\nu=1 / 3$. The solid line refers to the refined theory, the long-dashed line to thin shell theory, and the short-dashed line to the Reissner-Wan thin shell theory.

the differences between the refined and thin shell values are almost entirely due to errors of relative order $O(\epsilon)$ present in the thin shell values. Thus classical thin shell theory is no longer appropriate when $\epsilon$ is as large as $1 / 10$. However, the ReissnerWan theory gives values that are considerably closer to the "true" values than those of classical thin shell theory. The Reissner-Wan theory is not a two-term theory in the asymptotic sense, but nevertheless the values that it predicts are quite close to those predicted by our asymptotically correct two-term theory. The allowance for transverse shear deformation evidently enables the Reissner-Wan theory to be used for thicker shells than the classical theory.

It should be noted that the observation above on the accuracy of the ReissnerWan solution is consistent with the solution behavior obtained by Simmonds [15] for flat plates. In an analysis of three-dimensional plates under concentrated loads, Simmonds found that, near a concentrated load, classical plate theory gave better results for certain variables than did Reissner's refined plate theory; far from the load, Reissner's theory was better. As pointed out in the discussion following (4.15), our two-term outer asymptotic solution of the three-dimensional elasticity problem, which gives rise to the shell quantities (4.5)-(4.9), is valid at a distance from the load point not small compared to $\sqrt{R h}$. In a small neighborhood within a radius of order $O(h)$ of the load point (corresponding to Simmonds's small neighborhood near a concentrated load applied to a flat plate), the shell behavior should be derived from the inner asymptotic solution of the three-dimensional elasticity problem. Nothing is said about this inner solution in the present paper, and the corresponding shell behavior in this region may well be quite different from the Reissner-Wan solution.

Appendix A. The shell (or S-) eigenfunction. The eigenvalues of the axisymmetric eigenfunctions of the spherical shell satisfy the equation

$$
\left[\frac{\sinh \gamma \beta}{\sinh \gamma}\right]^{2}=\beta^{2}\left[\frac{\beta^{4}-\frac{5}{2} \beta^{2}+\frac{73}{16}-4 \nu^{2}}{\beta^{4}+\beta^{2}\left[4\left(1-\nu^{2}\right)-\frac{5}{2}\right]+\frac{9}{16}}\right]
$$


where

$$
\gamma=\ln \left(\frac{1+\epsilon}{1-\epsilon}\right)
$$

The eigenvalue $\beta(\epsilon, \nu)$ governing the decay of the S-eigenfunction is given by

$$
\beta=\frac{(1+i) \omega}{\epsilon^{\frac{1}{2}}}\left[1-i\left(\frac{1+24 \nu^{2}}{80 \omega^{2}}\right) \epsilon+O\left(\epsilon^{2}\right)\right]
$$

as $\epsilon \rightarrow 0$, where the constant $\omega$ is given by

$$
\omega=\left[\frac{3}{4}\left(1-\nu^{2}\right)\right]^{1 / 4} \text {. }
$$

We denote the S-eigenfunction by $\left\{\boldsymbol{\tau}^{S}, \mathbf{u}^{S}\right\}$. As $\epsilon \rightarrow 0$, the components of $\left\{\boldsymbol{\tau}^{S}, \mathbf{u}^{S}\right\}$ are given by the asymptotic formulae below. In these formulae, $\xi$ is the dimensionless radial coordinate given by $r=R(1+\epsilon \xi)$.

\section{Displacements.}

$$
u_{r}^{S}(\xi, \phi)=v_{r}(\xi) P_{\beta-\frac{1}{2}}(\cos \phi),
$$

where

$$
\begin{aligned}
& R^{-1} \Re\left(v_{r}\right)=2-2 \nu \xi \epsilon+O\left(\epsilon^{2}\right), \\
& R^{-1} \Im\left(v_{r}\right)=-\frac{2 \nu \omega^{2} \xi^{2}}{1-\nu} \epsilon+O\left(\epsilon^{2}\right) . \\
& u_{\phi}^{S}(\xi, \phi)=v_{\phi}(\xi) \frac{d}{d \phi} P_{\beta-\frac{1}{2}}(\cos \phi),
\end{aligned}
$$

where

$$
\begin{aligned}
& R^{-1} \Re\left(v_{\phi}\right)=-2 \xi \epsilon+\frac{\left(2 \nu^{2}-20 \nu-2+15 \nu(1-\nu) \xi^{2}\right)}{15(1-\nu)} \epsilon^{2}+O\left(\epsilon^{3}\right), \\
& R^{-1} \Im\left(v_{\phi}\right)=-\frac{4 \omega^{2}}{3(1-\nu)} \epsilon+\frac{2 \omega^{2} \xi\left(4-(2-\nu) \xi^{2}\right)}{3(1-\nu)} \epsilon^{2}+O\left(\epsilon^{3}\right) .
\end{aligned}
$$

\section{Stresses.}

$$
\tau_{r r}^{S}(\xi, \phi)=\sigma_{r r}(\xi) P_{\beta-\frac{1}{2}}(\cos \phi),
$$

where

$$
\begin{aligned}
& E^{-1} \Re\left(\sigma_{r r}\right)=-\xi\left(1-\xi^{2}\right) \epsilon+O\left(\epsilon^{2}\right), \\
& E^{-1} \Im\left(\sigma_{r r}\right)=-\frac{2 \omega^{2}\left(1-\xi^{2}\right)}{1-\nu} \epsilon+O\left(\epsilon^{2}\right) .
\end{aligned}
$$

$$
\tau_{r \phi}^{S}(\xi, \phi)=\sigma_{r \phi}(\xi) \frac{d}{d \phi} P_{\beta-\frac{1}{2}}(\cos \phi),
$$


where

$$
\begin{aligned}
& E^{-1} \Re\left(\sigma_{r \phi}\right)=\frac{\left[3+15 \nu+2 \nu^{2}-5\left(1-\nu^{2}\right) \xi^{2}\right]\left(1-\xi^{2}\right)}{10\left(1-\nu^{2}\right)} \epsilon^{2}+O\left(\epsilon^{3}\right), \\
& E^{-1} \Im\left(\sigma_{r \phi}\right)=\frac{2 \omega^{2}\left(1-\xi^{2}\right)}{1-\nu^{2}} \epsilon-\frac{2 \omega^{2}(7+\nu) \xi\left(1-\xi^{2}\right)}{3\left(1-\nu^{2}\right)} \epsilon^{2}+O\left(\epsilon^{3}\right) . \\
& \tau_{\phi \phi}^{S}(\xi, \phi)=\sigma_{\phi \phi}^{(1)}(\xi) P_{\beta-\frac{1}{2}}(\cos \phi)+\sigma_{\phi \phi}^{(2)}(\xi) \cot \phi \frac{d}{d \phi} P_{\beta-\frac{1}{2}}(\cos \phi),
\end{aligned}
$$

where

$$
\begin{gathered}
E^{-1} \Re\left(\sigma_{\phi \phi}^{(1)}\right)=\frac{\xi\left[18+5 \nu-3 \nu^{2}-10\left(1-\nu^{2}\right) \xi^{2}\right]}{5\left(1-\nu^{2}\right)} \epsilon+O\left(\epsilon^{2}\right), \\
E^{-1} \Im\left(\sigma_{\phi \phi}^{(1)}\right)=\frac{4 \omega^{2} \xi}{1-\nu^{2}}+\frac{2 \omega^{2}\left[\nu-3(2+\nu) \xi^{2}\right]}{3\left(1-\nu^{2}\right)} \epsilon+O\left(\epsilon^{2}\right), \\
E^{-1} \Re\left(\sigma_{\phi \phi}^{(2)}\right)=\frac{2 \xi}{1+\nu} \epsilon+\frac{2+20 \nu-2 \nu^{2}-15(2+\nu)(1-\nu) \xi^{2}}{15\left(1-\nu^{2}\right)} \epsilon^{2}+O\left(\epsilon^{3}\right), \\
E^{-1} \Im\left(\sigma_{\phi \phi}^{(2)}\right)=\frac{4 \omega^{2}}{3\left(1-\nu^{2}\right)} \epsilon+\frac{2 \omega^{2} \xi\left[-6+(2-\nu) \xi^{2}\right]}{3\left(1-\nu^{2}\right)} \epsilon^{2}+O\left(\epsilon^{3}\right) . \\
\tau_{\theta \theta}^{S}(\xi, \phi)=\sigma_{\theta \theta}^{(1)}(\xi) P_{\beta-\frac{1}{2}}(\cos \phi)+\sigma_{\theta \theta}^{(2)}(\xi) \cot \phi \frac{d}{d \phi} P_{\beta-\frac{1}{2}}(\cos \phi),
\end{gathered}
$$

where

$$
\begin{aligned}
& E^{-1} \Re\left(\sigma_{\theta \theta}^{(1)}\right)=2+\frac{\xi\left[-10+3 \nu+15 \nu^{2}+12 \nu^{3}-5 \nu\left(1-\nu^{2}\right) \xi^{2}\right]}{5\left(1-\nu^{2}\right)} \epsilon+O\left(\epsilon^{2}\right) \\
& E^{-1} \Im\left(\sigma_{\theta \theta}^{(1)}\right)=\frac{4 \nu \omega^{2} \xi}{1-\nu^{2}}-\frac{2 \nu \omega^{2}\left[3+2 \nu+3(2+\nu) \xi^{2}\right]}{3\left(1-\nu^{2}\right)} \epsilon+O\left(\epsilon^{2}\right)
\end{aligned}
$$

and

$$
E^{-1} \sigma_{\theta \theta}^{(2)}=-E^{-1} \sigma_{\phi \phi}^{(2)} .
$$

Note. When $|\beta|$ is large (in accordance with (A.3) as $\epsilon \rightarrow 0$ ) and when $\phi$ is bounded away from 0 and $\pi$, we have that

$$
\begin{aligned}
P_{\beta-\frac{1}{2}}(\cos \phi) & \sim\left(\frac{i}{2 \pi \beta \sin \phi}\right)^{1 / 2} e^{-i \beta \phi}, \\
\frac{d}{d \phi} P_{\beta-\frac{1}{2}}(\cos \phi) & \sim-i \beta P_{\beta-\frac{1}{2}}(\cos \phi) .
\end{aligned}
$$

Equations (A.26), (A.27) display the exponential variation of $\left\{\boldsymbol{\tau}^{S}, \mathbf{u}^{S}\right\}$ with $\phi$.

Appendix B. The three-dimensional membrane state. The cap (and complementary cap) eigenfunctions associated with the roots of the eigenvalue equation (A.1) are not sufficient to expand the required solution $\left\{\boldsymbol{\tau}^{E X}, \mathbf{u}^{E X}\right\}$. This is because all these eigenfunctions correspond to zero resultant applied load in the $z$-direction, which is clearly not true for $\left\{\boldsymbol{\tau}^{E X}, \mathbf{u}^{E X}\right\}$. The expansion set is completed by the addition of a suitable "membrane state," the simplest choice for which is that given by Gregory, Milac, and Wan [6], which is reproduced below. In these formulae, $\xi$ is the dimensionless radial coordinate defined by $r=R(1+\xi \epsilon)$. 


\section{Displacements.}

$$
\begin{gathered}
R^{-1} u_{r}^{M}=\cos \phi \ln \left(\cot \frac{1}{2} \phi\right)-1 . \\
R^{-1} u_{\phi}^{M}=-\sin \phi \ln \left(\cot \frac{1}{2} \phi\right)-\cot \phi .
\end{gathered}
$$

\section{Stresses.}

$$
\begin{gathered}
E^{-1} \tau_{r r}^{M}=0 . \\
E^{-1} \tau_{r \phi}^{M}=0 . \\
E^{-1} \tau_{\phi \phi}^{M}=(1+\nu)^{-1}(1+\xi \epsilon)^{-1} \csc ^{2} \phi . \\
E^{-1} \tau_{\theta \theta}^{M}=-(1+\nu)^{-1}(1+\xi \epsilon)^{-1} \csc ^{2} \phi . \\
\text { REFERENCES }
\end{gathered}
$$

\section{REFERENCES}

[1] J.G. BerRY, On thin hemispherical shells subjected to concentrated edge moments and forces, in Proc. Second Midwest Conference on Solid Mechanics, Purdue University, 1955, pp. $25-44$.

[2] W. FlügGe, Stresses in Shells, 2nd ed., Springer, New York, 1973.

[3] Y.C. Fung, Foundations of Solid Mechanics, Prentice-Hall, Englewood Cliffs, NJ, 1965.

[4] R.D. Gregory, The semi-infinite strip $x \geq 0,-1 \leq y \leq 1$; completeness of the PapkovichFadle eigenfunctions when $\phi_{x x}(0, y), \phi_{y y}(0, y)$ are prescribed, J. Elasticity, 10 (1980), pp. $57-80$.

[5] R.D. GREGory, The traction boundary value problem for the elastostatic semi-infinite strip; existence of solution, and completeness of the Papkovich-Fadle eigenfunctions, J. Elasticity, 10 (1980), pp. 295-327.

[6] R.D. Gregory, T.I. Milac, And F.Y.M. Wan, The axisymmetric deformation of a thin, or moderately thick, elastic spherical cap, Stud. Appl. Math., 100 (1998), pp. 67-94.

[7] W.T. KoIter, A spherical shell under point loads at its poles, in Progress in Applied Mechanics (Prager Anniversary Volume), Macmillan, New York, 1963, pp. 155-170.

[8] W. Leutert, The heavy sphere supported by a concentrated force, Pacific J. Math., 1 (1951), pp. $97-101$.

[9] A.E. LuR'E, Equilibrium of an elastic symmetrically loaded spherical shell, Prikl. Mat. Mekh., 7 (1943), pp. 393-404 (in Russian).

[10] A.E. LuR'E, Three-Dimensional Problems of the Theory of Elasticity, Interscience, New York, 1964.

[11] T.I. Milac, The Linear Elastostatics of Spherical Shells, Ph.D. Thesis, University of Washington, Seattle, Washington, 1995.

[12] F.W.J. Olver, Asymptotics and Special Functions, Academic Press, New York, 1974.

[13] E. ReIsSner, Stresses and small displacements of shallow spherical shells I, II, J. Math. Phys., 25 (1947), pp. 80-85, 279-300.

[14] E. REISSNER AND F.Y.M. WAN, Rotationally symmetric stress and strain in shells of revolution, Stud. Appl. Math., 48 (1969), pp. 1-17.

[15] J.G. Simmonds, Pointwise errors in the classical and in Reissner's linear theory of plates, especially for concentrated loads, J. Elasticity, 23 (1990), pp. 219-232.

[16] E. Sternberg and F. Rosenthal, The elastic sphere under concentrated loads, J. Appl. Mech., 19 (1952), pp. 413-421.

[17] T.V. Vilenskaia And I.I. Vorovich, Asymptotic behavior of the solution of the problem of elasticity for a thin spherical shell, Prikl. Mat. Mekh., 30 (1966), pp. 278-295. 